Acta Zoológica Mexicana (nueva serie), Volumen 37, 1-19.

https://doi.org/10.21829/azm.2021.3712413

Artículo original

\title{
La Colección Entomológica del Instituto- Fundación Miguel Lillo, Tucumán, Argentina
}

\section{The Entomologic Collection of the Instituto- Fundación Miguel Lillo, Tucumán, Argentina}

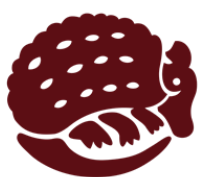

OPEN ACCESS

*Autor corresponsal:

Silvia Patricia Córdoba spcordoba@lillo.org.ar

Cita:

Córdoba, S. P., Pérez, E. C. (2021) La

Colección Entomológica del Instituto-Fundación Miguel Lillo,

Tucumán, Argentina. Acta Zoológica Mexicana (nueva serie),

37, 1-19.

10.21829/azm.2021.3712413 elocation-id: e3712413

Recibido: 08 mayo 2021 Aceptado: 04 agosto 2021 Publicado: 13 agosto 2021

\section{*Silvia Patricia CóRdoba, Emilia Constanza PÉREZ}

Instituto de Entomología, Fundación Miguel Lillo, Miguel Lillo 251 (4000), San Miguel de Tucumán, Argentina.

Editor responsable: Magdalena Cruz-Rosales

RESUMEN. La Colección Entomológica del Instituto-Fundación Miguel Lillo (IFML) conserva y custodia el material allí depositado con el fin de facilitar su estudio por parte de toda la comunidad científica actual y futura. Está constituida por la colección entomológica conservada en seco y en alcohol, en dos salones equipados para el resguardo y conservación del material. Atesora 6,500,000 ejemplares de los órdenes Blattodea, Coleoptera, Collembola, Dermaptera, Diptera, Embioptera, Ephemeroptera, Hemiptera, Hymenoptera, Isoptera, Mecoptera, Megaloptera, Lepidoptera, Neuroptera, Odonata, Orthoptera, Phthiraptera, Plecoptera, Psocoptera, Thysanoptera, Trichoptera y Zygentoma. La colección entomológica del I-FML preserva 11,216 ejemplares de la serie tipo, asignados a 2,185 especies, principalmente del Neotrópico, además de ejemplares de diversas partes del mundo. Es un referente de la biodiversidad de insectos neotropicales y ha sido fundada en el año 1944 a partir del material colectado por el Dr. Miguel Lillo. El objetivo del presente trabajo es dar a conocer de manera general, la organización, características, situación actual y perspectivas futuras de la colección entomológica del I-FML. Dada su importancia, tanto para la comunidad científica como para la sociedad en general, es necesario garantizar la conservación de los ejemplares allí depositados.

Palabras clave: América del Sur; región Neotropical; insectos; 
conservación; especímenes; biodiversidad

ABSTRACT. The Entomological Collection of the Miguel Lillo Foundation-Institute (IFML) preserves and guards the material deposited there, to facilitate study by the entire current and future scientific community. It holds 6,500,000 specimens of the orders Blattodea, Coleoptera, Collembola, Dermaptera, Diptera, Embioptera, Ephemeroptera, Hemiptera, Hymenoptera, Isoptera, Mecoptera, Megaloptera, Lepidoptera, Neuroptera, Odonata, Orthoptera, Phthiraptera, Plecoptera, Psocoptera, Thysanoptera, Trichoptera and Zygentoma. The entomological collection of the I-FML preserves 11,216 specimens of the types series assigned to 2,185 species, mainly from the Neotropic, in addition to specimens from various parts of the world. It is made up of the entomological collection preserved dry and in alcohol, in two rooms equipped for the protection and conservation of the material. It is a benchmark for the biodiversity of neotropical insects and was founded in 1944 based on the material collected by Dr. Miguel Lillo. The objective of this paper is to present in general way, the entomological collection of the I-FML, its organization, characteristics, current situation and future perspectives. Given its importance, both for the scientific community and for society in general, it is necessary to guarantee the conservation of the specimens deposited in it.

Key words: South America; Neotropical region; insects; conservation; specimens; biodiversity

\section{INTRODUCCIÓN}

Las colecciones biológicas representan el patrimonio natural de un país o región, constituyen un archivo histórico natural de utilidad múltiple donde la preservación de especímenes y su información asociada son la base de estudios taxonómicos, sistemáticos, ecológicos, filogenéticos, biogeográficos, de genética de poblaciones y conservación, formando parte fundamental en el conocimiento de la diversidad biológica y en el avance de las ciencias biológicas (Tobar, 2002).

La importancia de las colecciones biológicas radica en el aprovechamiento que la comunidad científica pueda hacer de ellas y en las consecuencias que se deriven de ahí. Brindan datos primarios que podrían ser usados en innumerables investigaciones posteriores y mantienen una relevancia histórica ya que constituyen la evidencia física sobre la que se generan múltiples publicaciones a lo largo del tiempo (Vargas, 2010). Han sido grandemente beneficiosas, tanto para el mundo natural como para la sociedad, desempeñando un papel vital en aspectos de salud humana (estudios de patógenos y vectores de enfermedades), monitoreo de cambios ambientales (seguimiento de contaminantes ambientales y cambio climático global) y, por supuesto, en el avance de las ciencias biológicas, incluyendo estudios de la biodiversidad y su pérdida (Páez, 2017).

Las colecciones de historia natural tienen el potencial de promover poderosos enfoques interdisciplinarios e históricos para estudiar patógenos zoonóticos emergentes (Joffrin et al., 2020). Los especímenes archivados representan una vasta infraestructura de biodiversidad, en gran parte sin explotar, que puede facilitar la identificación de patógenos y la comprensión de la ecología del 
hospedador, la inmunología, la coevolución y la dinámica hospedador-patógeno, que pueden ser ingredientes necesarios para la predicción de enfermedades (Thompson, 2021).

El Instituto de Entomología de la Fundación Miguel Lillo (I-FML) resguarda una importante colección entomológica, reconocida como la tercera en importancia de Argentina (Claps et al., 2013) y como una de las más prestigiosas de América del Sur. En el Instituto se desarrollan diferentes líneas de investigación sobre estudios taxonómicos, ecológicos, biológicos, de plagas, control biológico y de distribución de los insectos, que permiten un continuo enriquecimiento de la colección. Investigadores pertenecientes al Instituto Superior de Entomología "Dr. Abraham Willink" (INSUE), a la Facultad de Ciencias Naturales de la Universidad Nacional de Tucumán (CSNAT, UNT) y al Consejo Nacional de Investigaciones Científicas, Unidad Ejecutora Lillo (CONICET, UEL), contribuyen con su acrecentamiento y ordenamiento.

La colección entomológica del Instituto-Fundación Miguel Lillo (acrónimo: IFML) tiene como principal objetivo preservar y custodiar el material allí depositado y facilitar su acceso a toda la comunidad científica actual y futura. Es un acervo de la biodiversidad de insectos neotropicales, donde la mayoría de los especímenes fueron recolectados en Argentina y países limítrofes; cuenta, además, con ejemplares de diversos lugares del mundo. Esta colección se constituyó en 1944 a partir de las colecciones realizadas por el Dr. Miguel Lillo y de material donado por Rodolfo Schreiter (ver historia en Aranda et al., 2016). Posteriormente, como resultado de donaciones, canjes y colectas, esta colección se fue incrementando hasta la actualidad. En los últimos 77 años, científicos de la Fundación Miguel Lillo e investigadores de otras Instituciones (Instituto Superior de Entomología, Facultad de Ciencias Naturales e Instituto Miguel Lillo, Universidad Nacional de Tucumán, Consejo Nacional de Investigaciones Científicas y Técnicas, Estación Experimental Agroindustrial Obispo Colombres, Instituto Nacional de Tecnología Agropecuaria, etc.) han trabajado incesantemente para resguardar e incrementar esta colección, lo que indica que está en constante progreso y dinamismo.

El objetivo del presente trabajo es dar a conocer, de manera general, la organización, características, situación actual y perspectivas a futuro de la colección del I-FML, colección que por su historia e importancia es considerada como referente en estudios de biodiversidad del noroeste de Argentina y países colindantes.

\section{MATERIALES Y MÉTODOS}

La Colección Entomológica del I-FML se encuentra organizada en la Colección Entomológica Conservada en Seco y la Conservada en Alcohol, en dos salones separados, ubicados en el tercer piso, del edificio de Zoología de la FML.

La Colección Entomológica Conservada en Seco contiene 312 armarios metálicos (Fig. 1) de los cuales, 270 contienen ejemplares montados en alfileres, seis corresponden a los que albergan los ejemplares de la serie tipo, y el resto a los ejemplares sin preparar (camas y sobres entomológicos). También cuenta con un armario de nidos del orden Hymenoptera. El material montado en alfileres (directamente en alfileres entomológicos, en punto, triángulo o en minucias) 
está resguardado en cajas entomológicas con tapas de vidrio herméticas, para evitar la acción del polvo, la humedad y las plagas de museos (insectos y hongos) (Fig. 2). El fondo de las cajas contiene un sustrato de polietileno expandido (plastazote) donde se encuentran pinchados los alfileres. Actualmente las cajas están siendo reacondicionadas con un sistema intercambiable de unidades de cartón con el plastazote en el fondo (de diferentes tamaños), las cuales facilitan la localización, el manejo del material en la tarea de digitalización de datos y finalmente su mejor preservación. Para el control de plagas de insectos y hongos, además de ser herméticas, las cajas están provistas de esquineros con naftalina y sílica gel, ambos efectivos para evitar plagas y moho (Fig. 2).

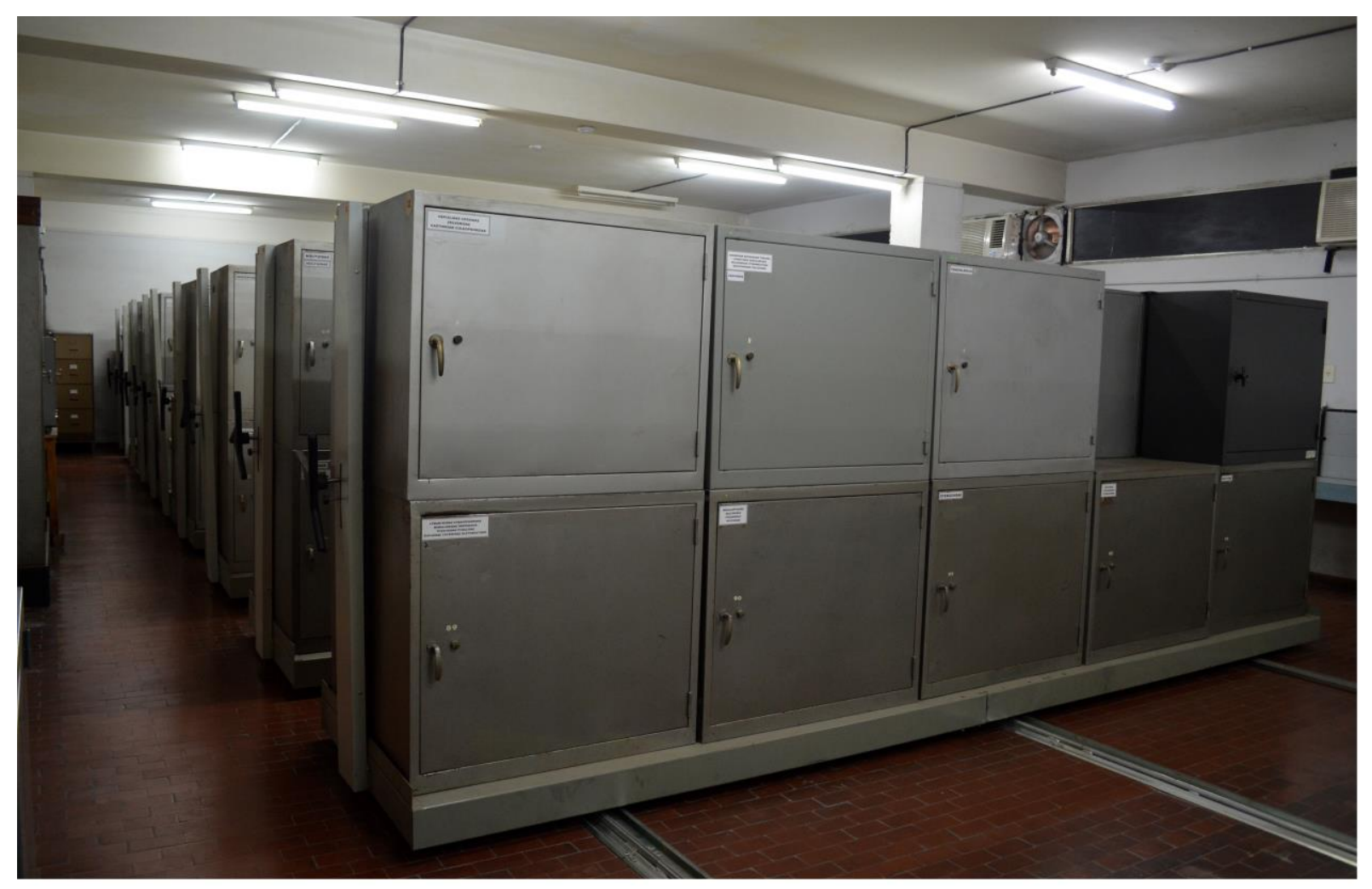

Figura 1. Salón de la Colección Entomológica Conservada en Seco del Instituto-Fundación Miguel Lillo, donde se aprecian los armarios metálicos y boggies.

Cada ejemplar contiene etiquetas blancas de papel libre de ácido, una con los datos de procedencia (información del origen del material), otra con la determinación taxonómica, una tercera con el nombre de la Institución y una cuarta que contiene el acrónimo y el número de catálogo, que se coloca a medida que se digitalizan los datos del ejemplar. En el caso del material tipo, además se agrega otra etiqueta roja para holotipos y alotipos, y una amarilla para los paratipos y sintipos (Fig. 3).

Cada caja tiene una etiqueta en el frente, donde se indican la o las familias que contiene. Las cajas entomológicas están apiladas dentro de los armarios, los cuales están organizados 
alfabéticamente y se encuentran numerados y con rótulos que señalan las familias que contienen. El material tipo se encuentra en armarios separados y especiales cercanos a las puertas de ingreso. La colección cuenta con un amplio salón equipado con boggies para el desplazamiento de los armarios y optimización del espacio (Fig. 1).

Para el mantenimiento de las condiciones ambientales óptimas, se dispone de ocho equipos de aire acondicionado programados, cuatro extractores y un deshumidificador. Las condiciones de temperatura y humedad (idealmente, entre 18 y $21^{\circ} \mathrm{C}$ de temperatura y entre el 40 y el $50 \%$ de humedad relativa ambiente) son visualizadas en un termo higrómetro digital. También cuenta con un freezer donde se coloca el material que proviene de colectas en el campo antes de su ingreso a la colección, y una estufa para el secado de material y de la sílica gel que será reutilizada. Para seguridad contra incendios, la colección está provista de sensores (detectores de humo con alarma) y matafuegos. Contiguo a la colección se encuentra un gabinete equipado (con instrumental entomológico, cámara de ablandamiento, microscopios estereoscópicos, computadoras, impresoras, entre otros), acondicionado para las tareas de preparación (separación, montaje, entre otras) y reacondicionamiento del material, donde trabajan cinco técnicos de apoyo especializados y con vasta experiencia en dichas tareas (Fig. 4).

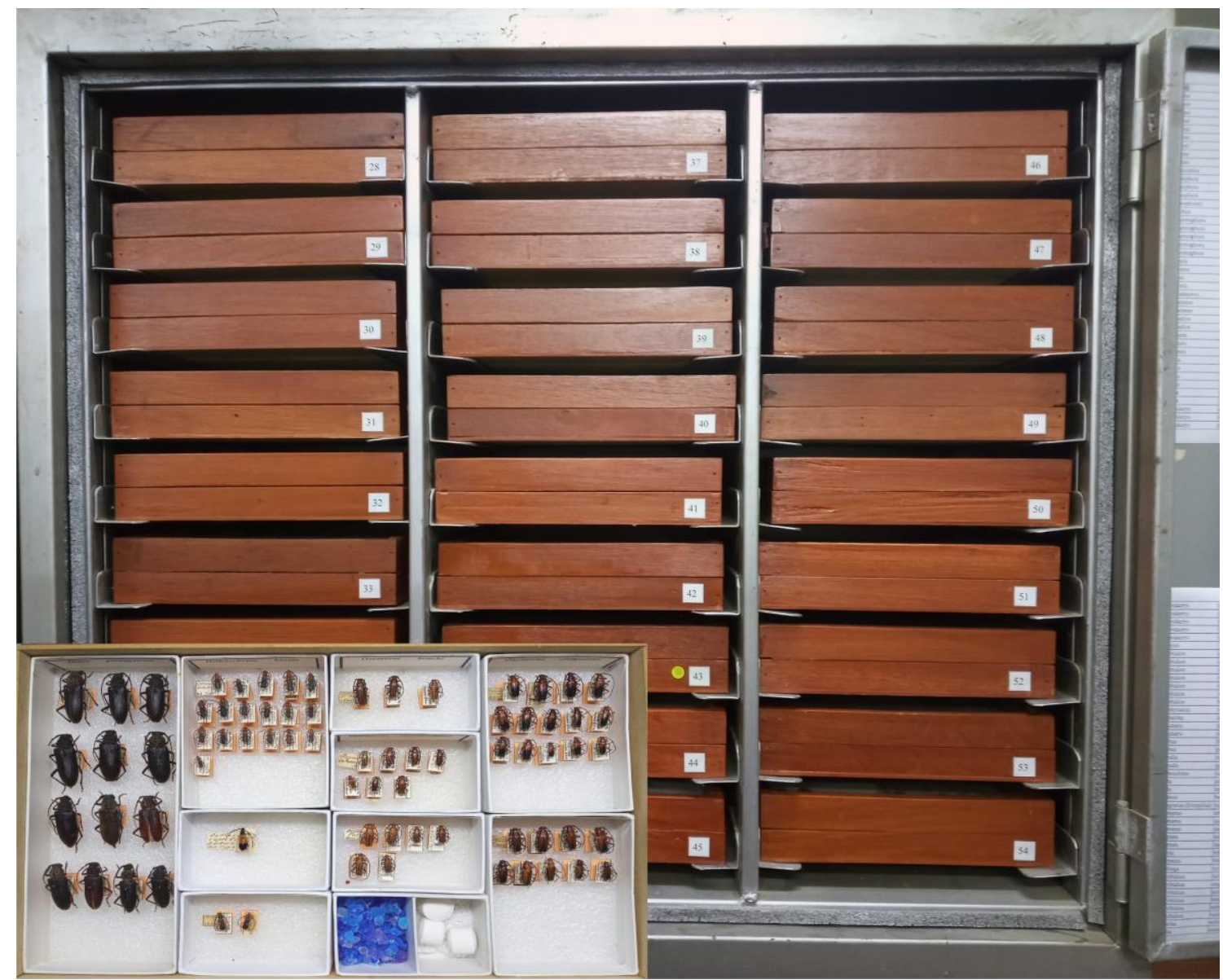

Figura 2. Detalle de un armario con caja entomológica de la Colección Entomológica Conservada en Seco del Instituto-Fundación Miguel Lillo. 
La colección se encuentra supervisada por la curadora Emilia C. Pérez (ecperez@lillo.org.ar). Además, para cada orden de insectos existe un encargado: José Ortega (Hymenoptera, Odonata, Ephemeroptera y Hemipetra), Francisco Sánchez (Coleoptera, neuropteroides y microlepidópteros), Alberto Rojas (Diptera, Orthoptera, Blattodea, Dermáptera, Phasmatodea, Mantodea, Isoptera, Thysanoptera, Plecoptera, Embioptera, Psocoptera y Trichoptera), Walter Lemos (Lepidoptera) y Rafael Palavecino (Hemiptera y Lepidoptera). Cada técnico revisa periódicamente el material a cargo para constatar el buen estado de conservación de los ejemplares, prepara el material que ingresa, facilita a los investigadores de la Institución y visitantes el material para estudio que fuera solicitado, entre otras tareas. Para la digitalización de los datos, se encuentran especializados, dos técnicos encargados específicamente de la confección de la base de datos: Gabriela Uzqueda y Federico Heredia. Esta base de datos contiene la totalidad de información que se conoce acerca de los ejemplares a fin de agilizar la consulta de las colecciones.

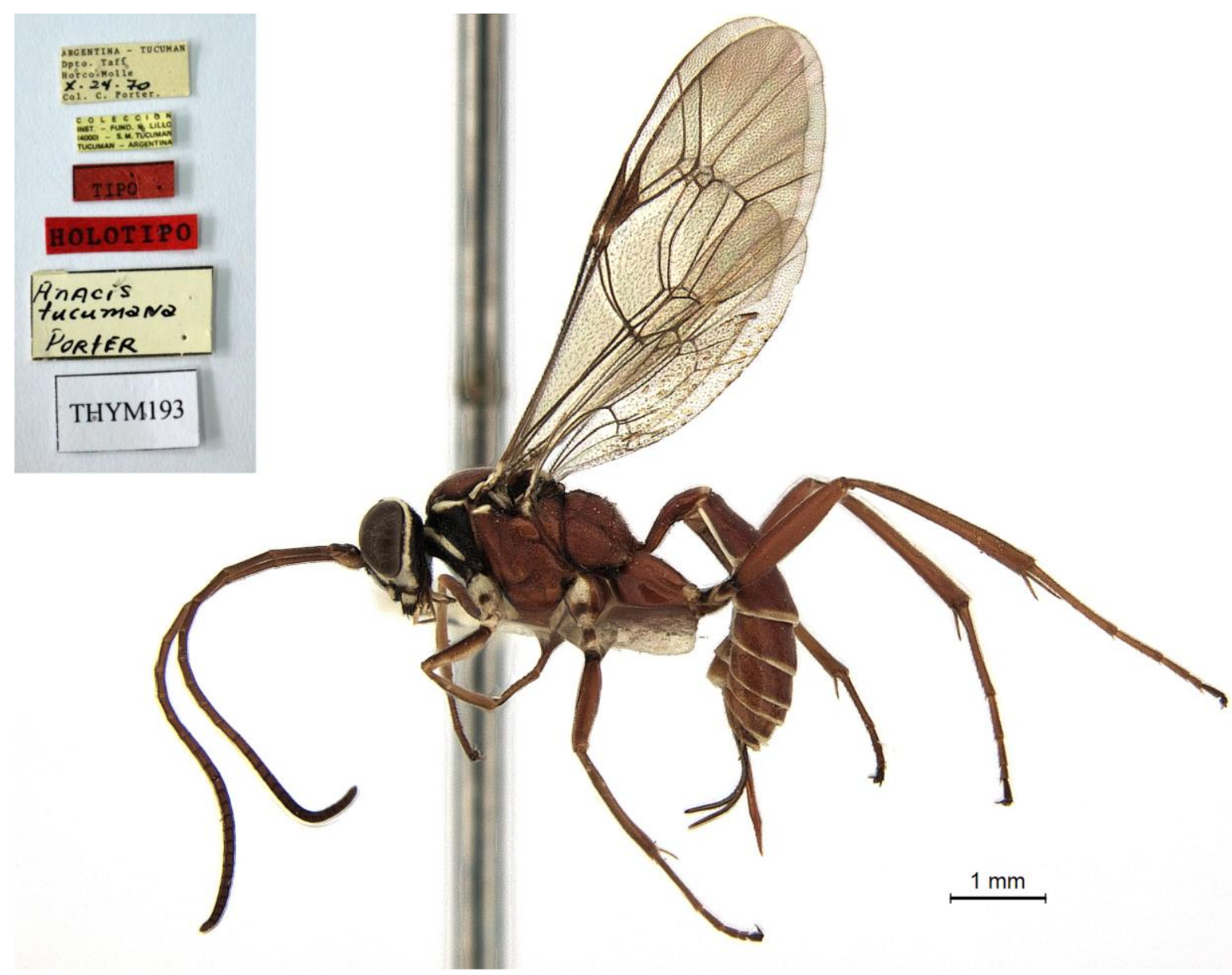

Figura 3. Ejemplar tipo de la Colección Entomológica Conservada en Seco del Instituto-Fundación Miguel Lillo. 
La Colección entomológica conservada en alcohol está organizada en 16 armarios, donde el material se mantiene en alcohol etílico (desde $70 \%$ hasta 96\%), para mantener la integridad del ADN de los especímenes, facilitando la utilización de éstos en trabajos moleculares y genéticos. Cada armario tiene un rótulo con el nombre respectivo de los órdenes que conserva, con números e ilustraciones realizadas a mano por el personal del Instituto de Iconografía de la FML (Fig. 5).

En cada armario, el material entomológico se encuentra depositado, en su mayoría, en tubos de Khan (de polipropileno) con tapón hermético y, a su vez, éstos están incluidos dentro de frascos de vidrio etiquetados que contienen el logo institucional, número y grupo taxonómico (Fig. 6). Estos frascos están ordenados alfabéticamente en los armarios y de acuerdo con su numeración. Cada tubo corresponde a un lote de ejemplares (determinado número de ejemplares con los mismos datos) y contienen también las etiquetas con la procedencia, determinación y otra con un número único y consecutivo (catalogación) (Fig. 7).

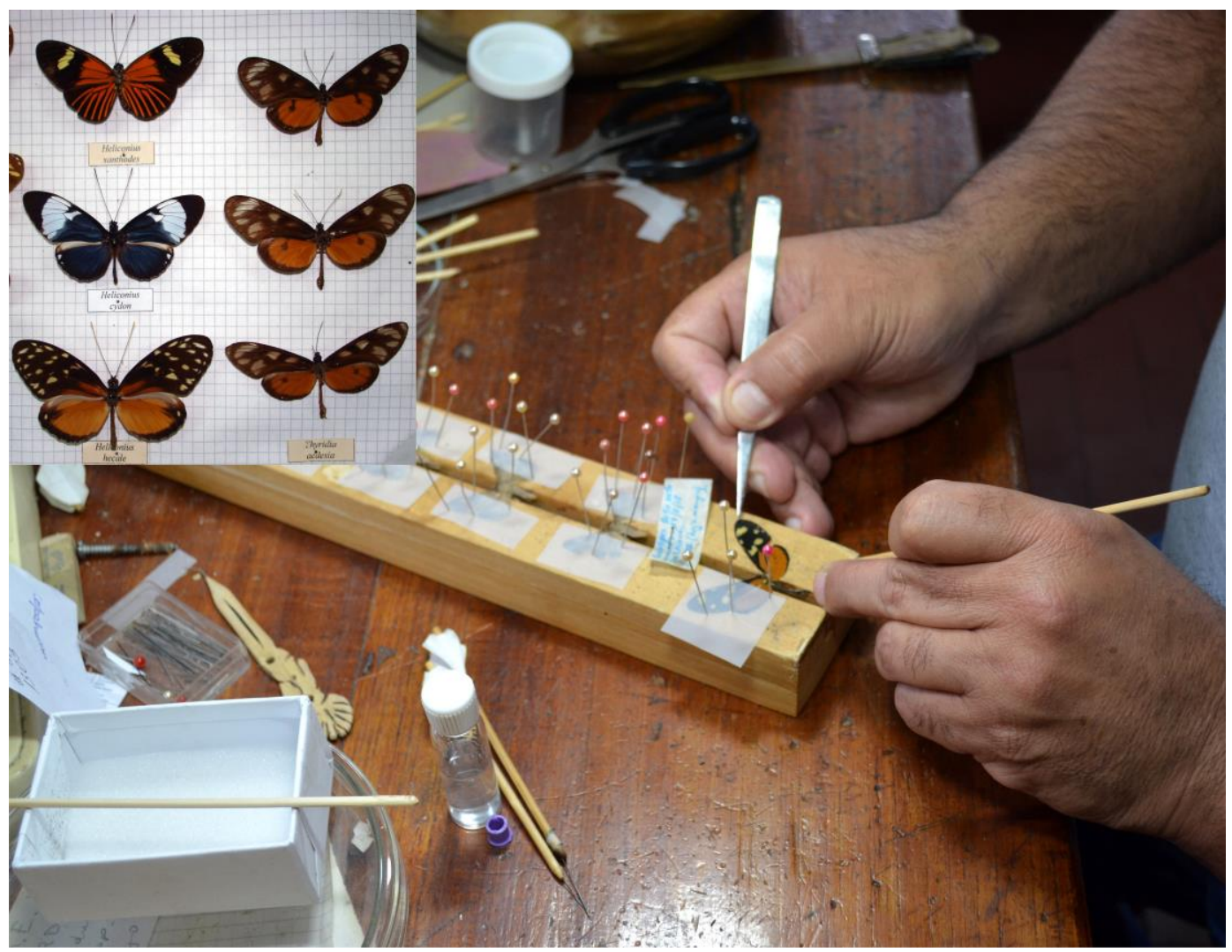

Figura 4. Preparación de ejemplares en seco, en el gabinete contiguo a la Colección Entomológica Conservada en Seco del Instituto-Fundación Miguel Lillo.

Esta colección está compuesta por un salón principal, donde se encuentran los armarios con material entomológico, entre los cuales está el más cercano a la puerta de ingreso que alberga los ejemplares de la serie tipo (Fig. 8). También hay dos armarios donde se guarda el instrumental y los elementos necesarios para la preservación de dicho material. 
Para mantener las condiciones de temperatura y humedad ideales (entre 18 y $21^{\circ} \mathrm{C}$ de temperatura y entre el 50 al $60 \%$ de humedad) el salón cuenta con un equipo de aire acondicionado, un deshumidificador portátil y termo higrómetro digital. Como sistemas de seguridad, la colección está provista de sensores contra incendios (detectores de humo con alarma), matafuegos y tres puertas de salida.

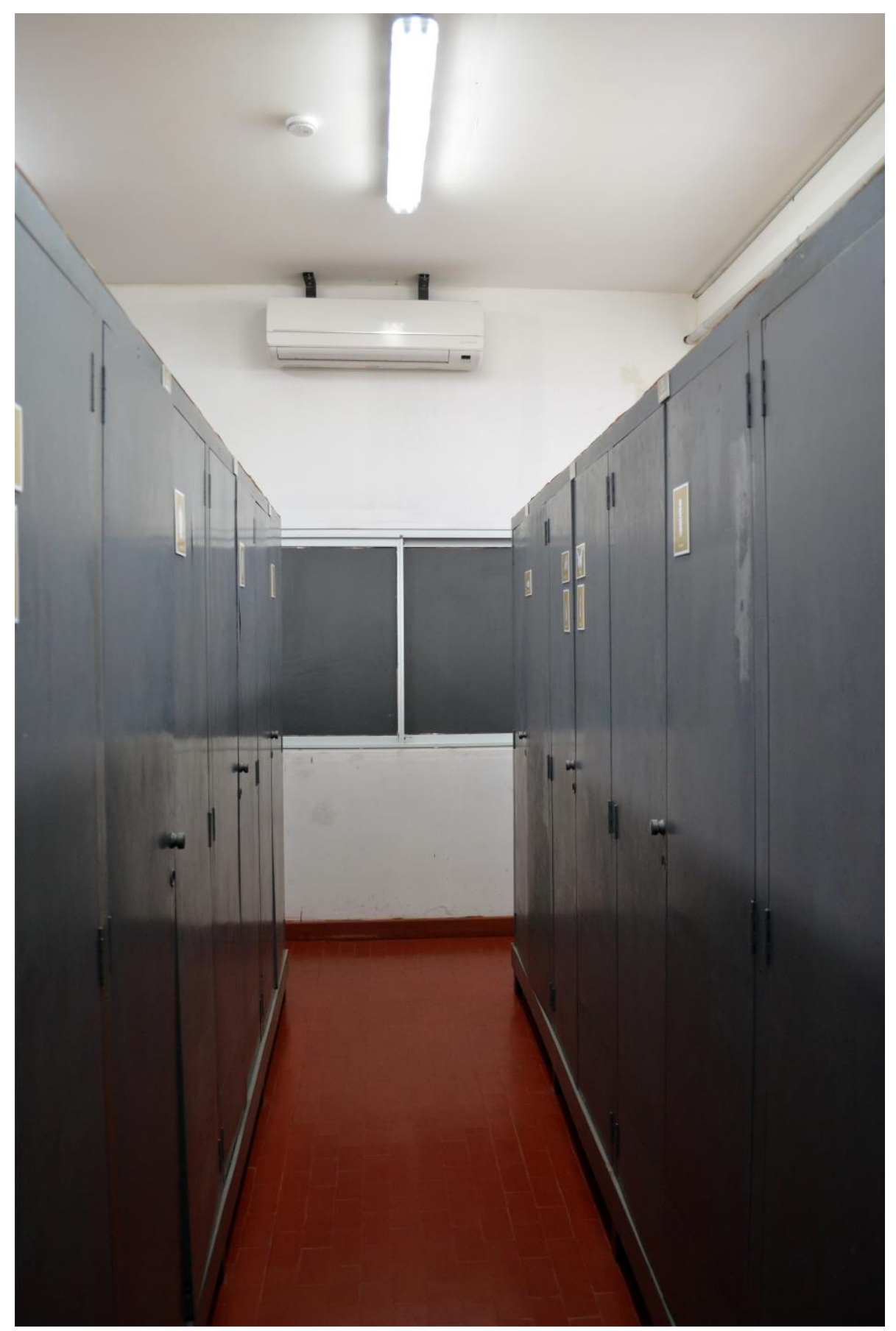

Figura 5. Salón principal de la Colección Entomológica Conservada en Alcohol del Instituto-Fundación Miguel Lillo. 


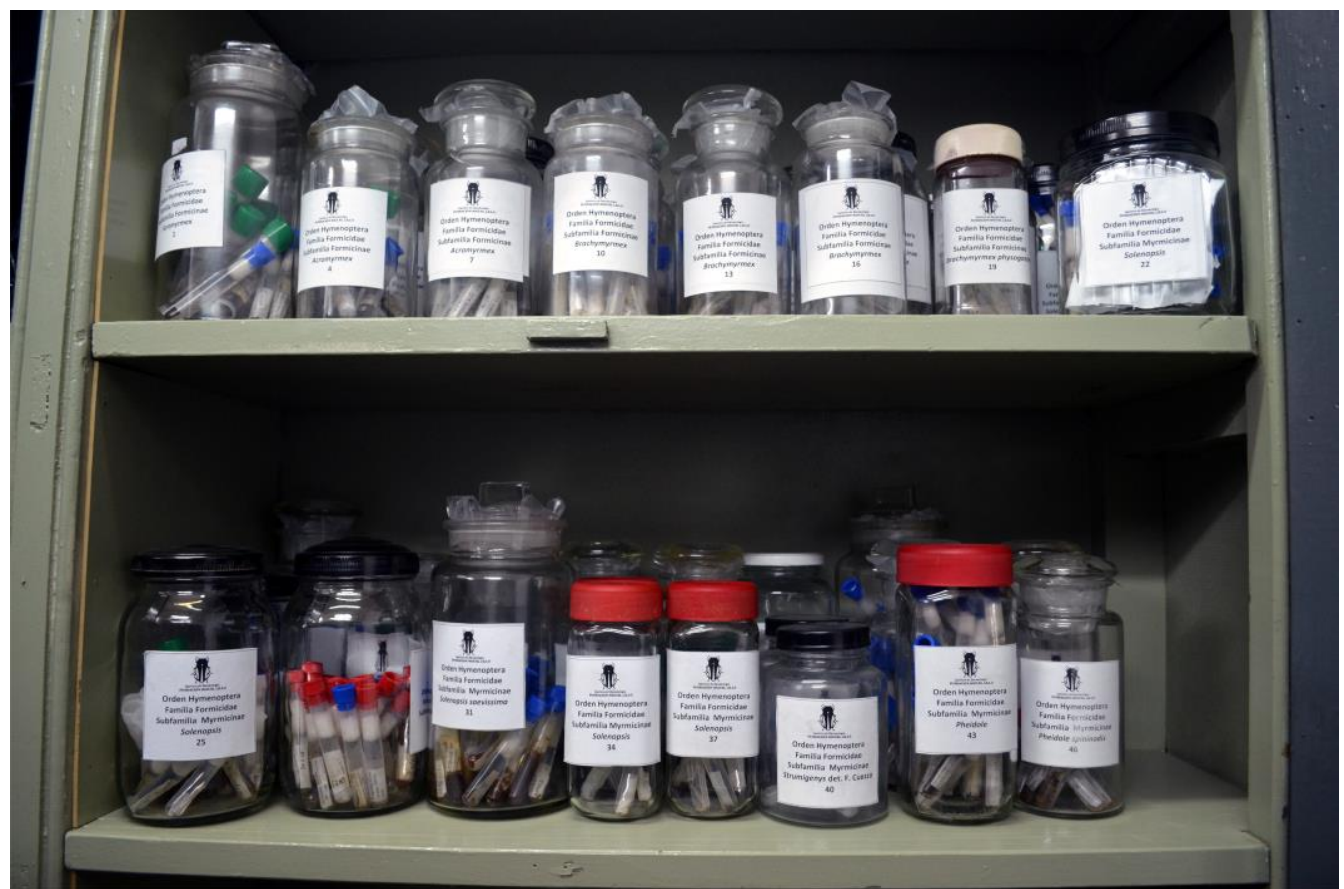

Figura 6. Frascos de vidrio etiquetados con tubos de khan en su interior, depositados en los armarios de la Colección Entomológica Conservada en Alcohol del Instituto-Fundación Miguel Lillo.

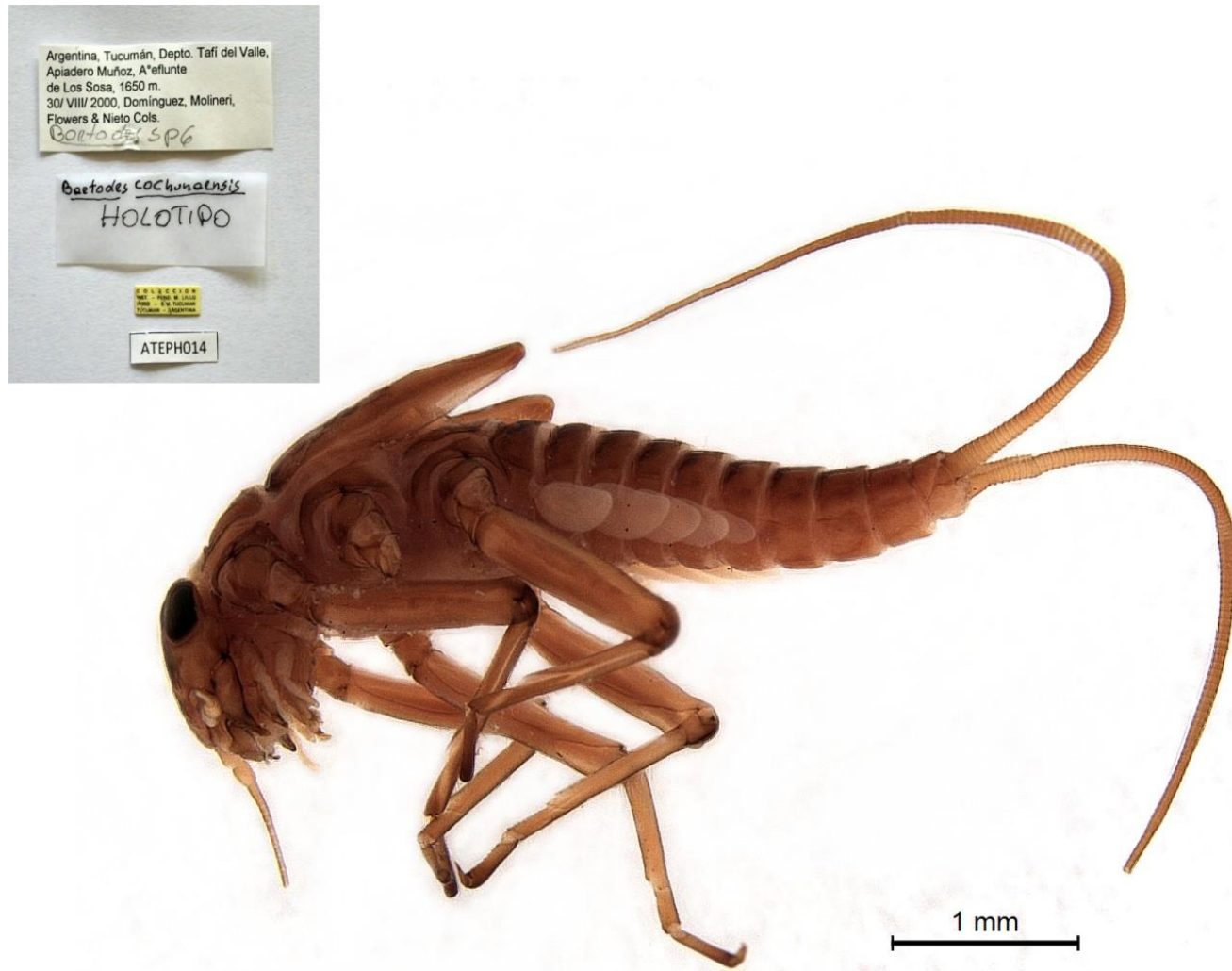

Figura 7. Ejemplar tipo de la Colección Entomológica Conservada en Alcohol del Instituto-Fundación Miguel Lillo. 


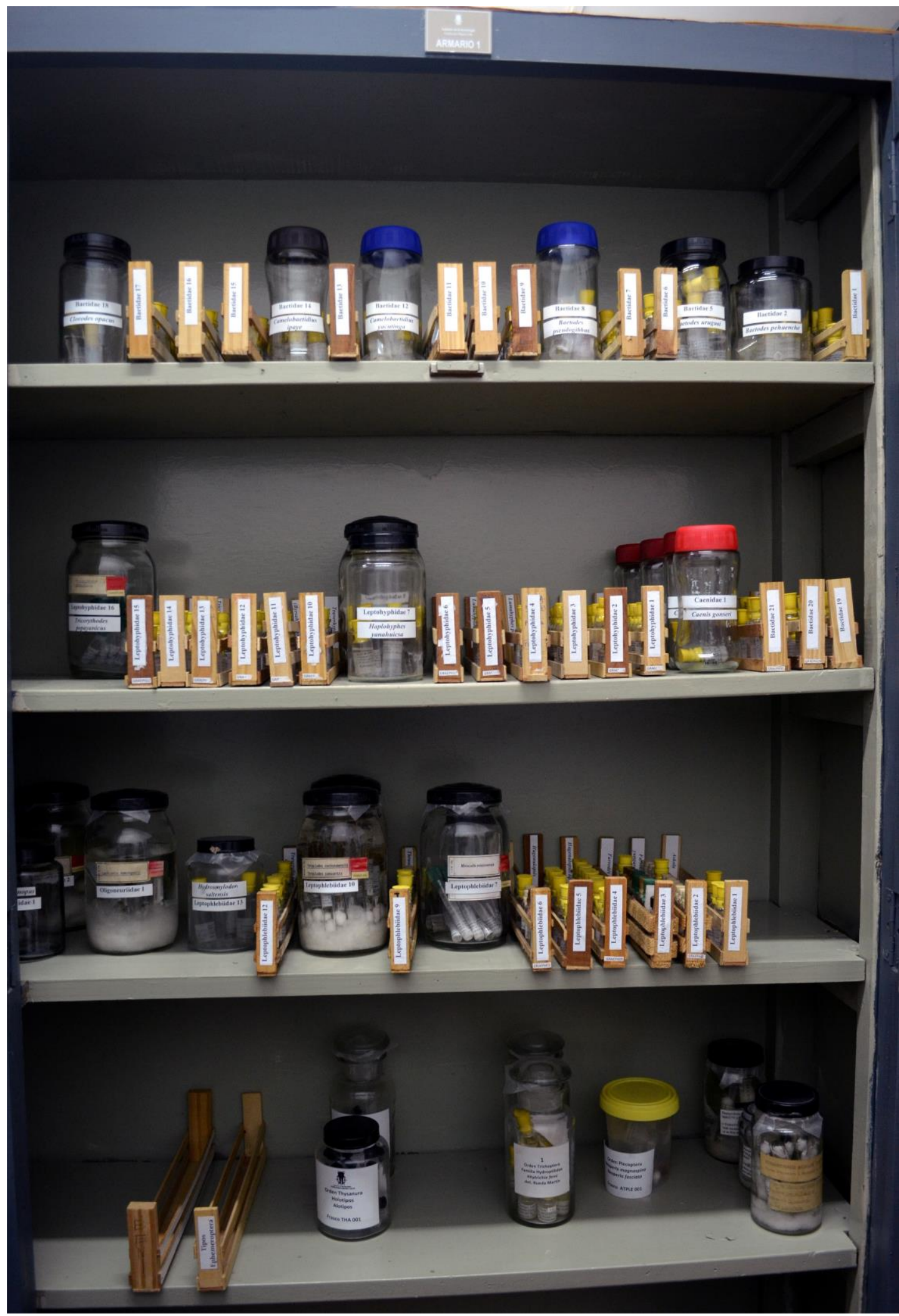

Figura 8. Armario de material tipo de la Colección Entomológica Conservada en Alcohol del InstitutoFundación Miguel Lillo. 
En su parte posterior se encuentra el gabinete donde se realizan los trabajos técnicos, los cuales incluyen separación, etiquetado y acondicionamiento de los ejemplares recolectados en el campo, como también se realizan las tareas de reacondicionamiento del material depositado, revisión del estado de conservación de los ejemplares y de las etiquetas, y renovación periódica del alcohol que lo mantiene. Asimismo, en el gabinete se encuentra el instrumental óptico (microscopio estereoscópico), computadora de escritorio y un destilador, con el que se obtiene el agua pura para preparar el alcohol que conservará el material.

La colección cuenta, además, con una base de datos digitalizada y otra en papel (carpetas en orden alfabético según el grupo taxonómico) del material depositado. Se encuentra a cargo de la responsable de dicha colección, Silvia P. Córdoba (spcordoba@lillo.org.ar) y como técnico de apoyo, Víctor Hugo Vera.

\section{RESULTADOS}

\section{Colección Entomológica Conservada en Seco}

El material entomológico conservado en seco comprende aproximadamente 4,500,000 especímenes, el mayor número corresponde a Hymenoptera, le siguen en orden de importancia (por el número de ejemplares) Coleoptera, Lepidoptera, Diptera y Hemiptera. El orden con mayor número de familias es Coleoptera, le siguen Diptera, Hymenoptera, Hemiptera, Lepidoptera, Trichoptera, Orthoptera, Odonata, Blattodea, Dermaptera, Mantodea, Neuroptera, Megaloptera, Plecoptera y Phasmathodea (Fig. 9).

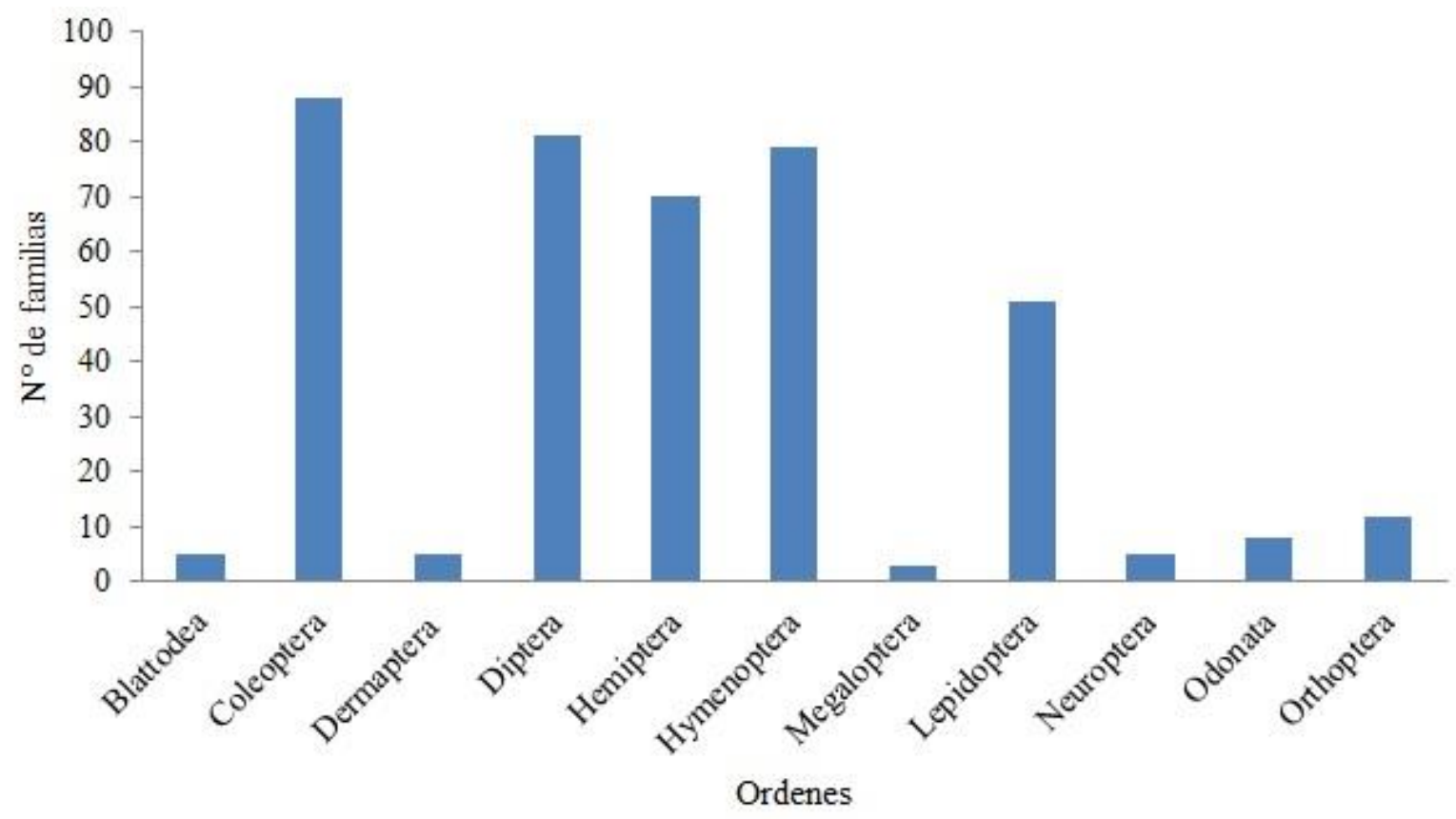

Figura 9. Número de las familias por cada orden de la Colección Entomológica Conservada en Seco del Instituto-Fundación Miguel Lillo. 
Además, cuenta con ejemplares de los órdenes Psocoptera y Strepsiptera sin determinar. El número de ejemplares de la serie tipo de la colección asciende a 7,170, asignados a 2,078 especies tipo, correspondientes a los órdenes Coleoptera, Diplura, Diptera, Hemiptera, Hymenoptera, Lepidoptera, Neuroptera, Odonnata, Phthiraptera y Zygentoma (Fig. 10).

También cuenta con cerca de 7,000 preparados microscópicos, la mayoría del orden Hemiptera (Homoptera), Phthiraptera, Coleoptera (genitalia) y algunos Lepidoptera (genitalia). Esta colección atesora ejemplares recolectados por el Dr. Miguel Lillo, tales como dos coleópteros de la familia Erotylidae, Iphiclus (Saccomorphus) klugi Lacordaire, 1842, de Las Cuchillas, Tucumán y Aegithus brunnipennis Lacordaire, 1842, de Metán, Salta, coleccionados por el Dr. Lillo en 1906. Los ejemplares de mayor antigüedad corresponden al himenóptero icneumónido Netelia sp., Tucumán, Siambón, de 1831, y a los coleópteros cerambícidos Ethemon basale (Burmeister, 1865) de 1900 y Trachyderes variegata Blackwelder, 1946 de 1902.

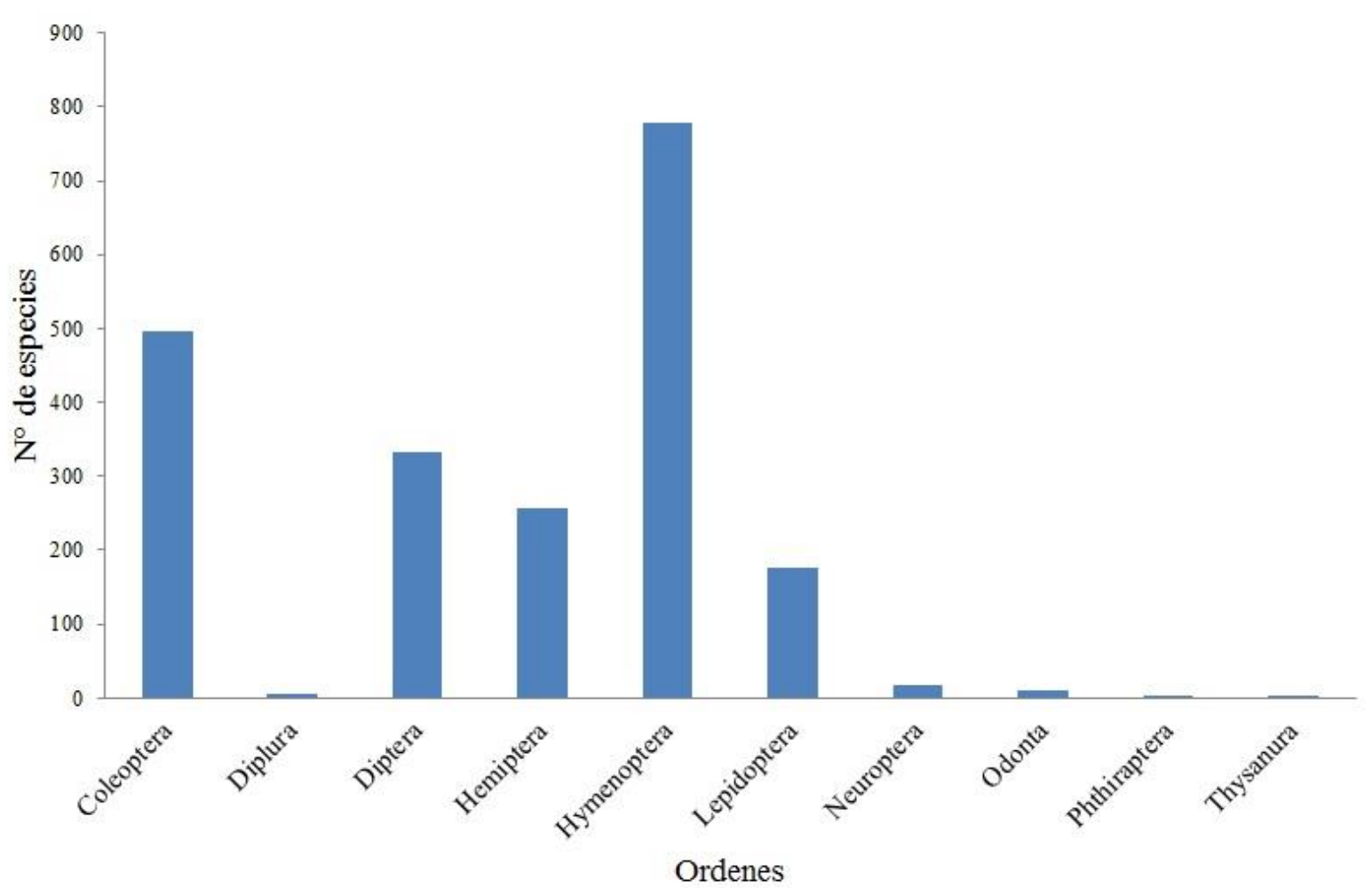

Figura 10. Número de especies tipo por cada orden de la Colección Entomológica Conservada en Seco del Instituto-Fundación Miguel Lillo.

\section{Colección Entomológica Conservada en Alcohol}

Acerva aproximadamente 2,000,000 de ejemplares, con representantes de casi todos los órdenes de insectos. El material depositado procede principalmente de Argentina, Bolivia, Uruguay, Paraguay y Brasil, con pocos representantes del resto del mundo. La mayor parte de los ejemplares corresponden al orden Hymenoptera, familia Formicidae, colectado por el Dr. Nicolás Kusnezov, 
quien trabajó en la Fundación Miguel Lillo desde 1947 hasta 1963, seguido por los órdenes Coleoptera, Diptera, Neuroptera, y material procedente de colectas en el marco de proyectos institucionales y de trampas Malaise. El orden con mayor número de familias en la colección es Diptera, le siguen Hemiptera, Coleoptera, Ephemeroptera, Hymenopetra, Isoptera, Neuroptera, Zygentoma, Lepidoptera, Trichoptera, Phthiraptera Blattodea, Dermaptera, Megaloptera, Orthoptera y Plecoptera (Fig. 11).

También se encuentran representados los órdenes Collembola, Embioptera, Homoptera (Hemiptera), Mecoptera, Psocoptera y Thysanoptera pero se encuentran aún sin determinar. Los ejemplares de la serie tipo pertenecen a los órdenes Diptera (familias Blephariceridae y Simulidae), Ephemeroptera (Baetidae, Caenidae, Leptohyphidae, Leptophlebiidae, Oligoneuriidae y Polymitarcyidae), Hymenoptera (Mymaridae), Isoptera (Kalotermitidae), Neuroptera (Coniopterygidae), Plecoptera (Gripopterygidae), Trichoptera (Hydrobiosidae y Hydroptilidae) y Zygentoma (Lepismatidae, Meinertellidae y Nicoletidae), los cuales suman 4,046 ejemplares, asignados a 107 especies (Córdoba, 2021) (Fig. 12). Los ejemplares de mayor antigüedad resguardados en la colección datan de 1922 y corresponden a 12 larvas de Lochmaea suturalis (Thompson, 1866) (Coleoptera, Chrysomelidae), 30/VII/1922, Alemania, A. Danft leg.

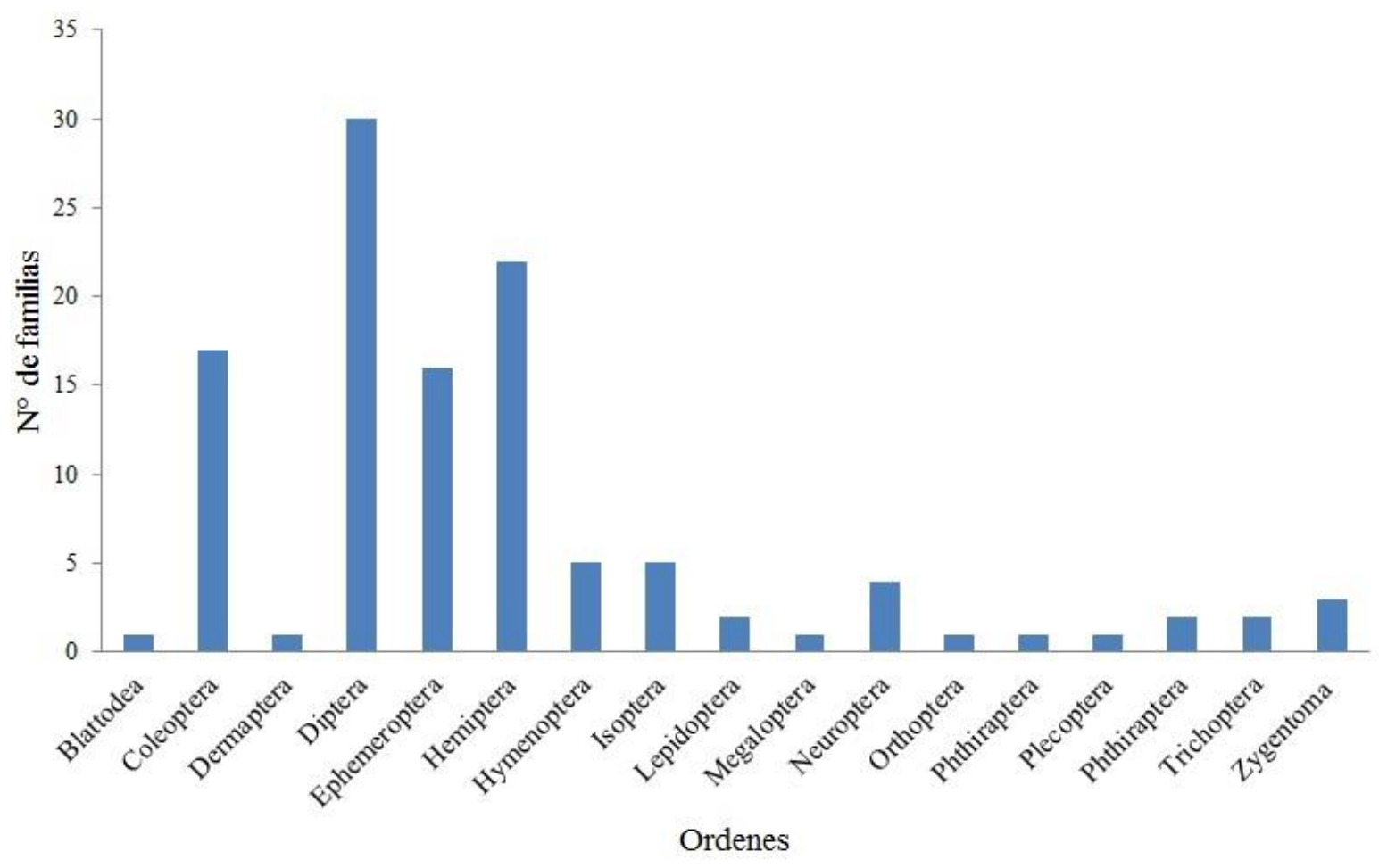

Figura 11. Número de familias por cada orden de la Colección Entomológica Conservada en Alcohol del Instituto-Fundación Miguel Lillo. 


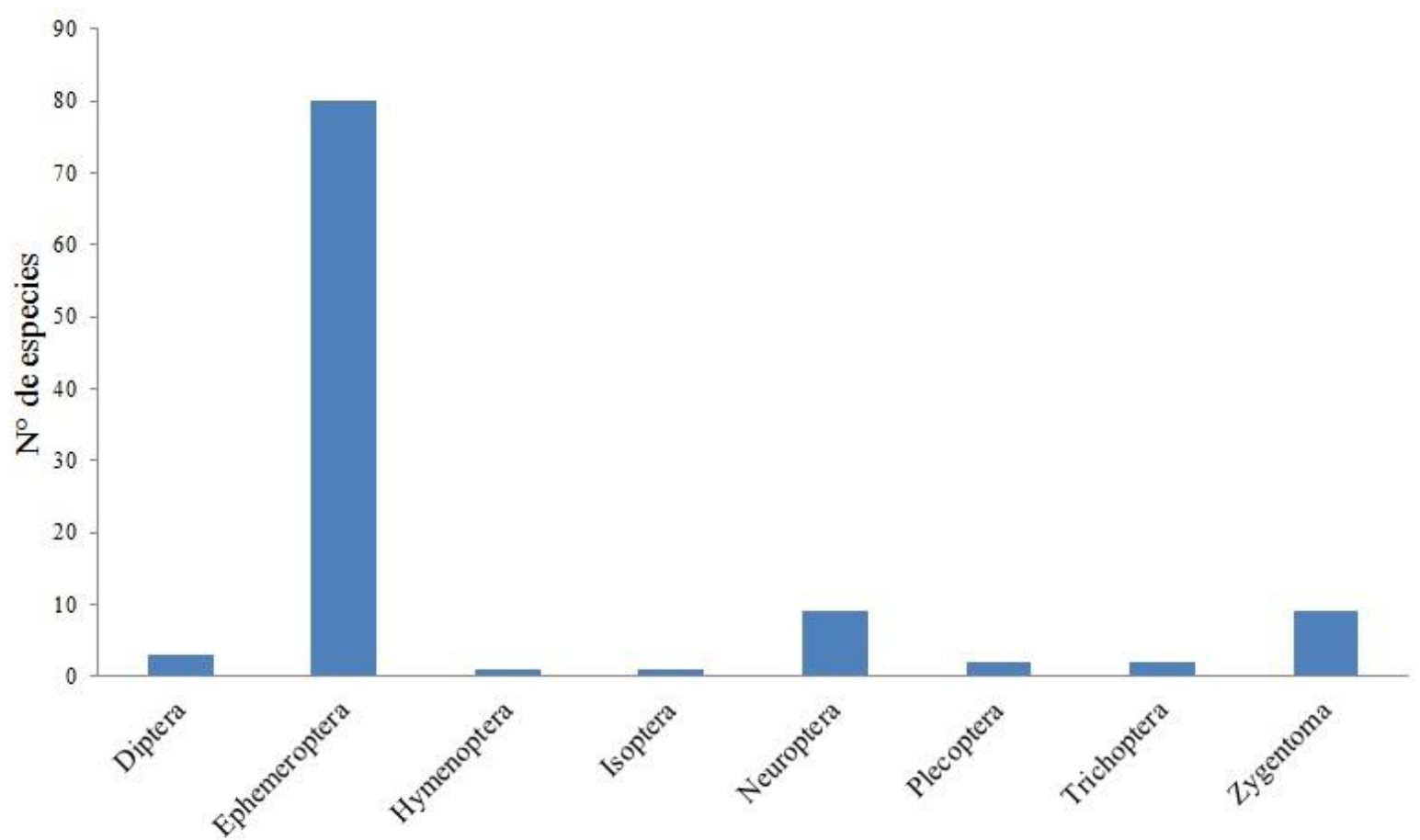

Ordenes

Figura 12. Número de especies tipo por cada orden de la Colección Entomológica Conservada en Alcohol del Instituto-Fundación Miguel Lillo.

\section{Préstamos y Digitalización}

La colección entomológica del I-FML mantiene un sistema de intercambio de material (préstamos, donaciones y canjes), que contribuye con su crecimiento y para el que existe un registro de consultas, préstamos y visitas a fin de mantener un control y evitar el extravío de material. Estos préstamos, donaciones y canjes están a cargo de la curadora de la colección y son autorizados previamente por la directora del Instituto de Entomología, la directora del Área de Zoología y por el director general de Investigaciones y se contemplan en el Reglamento Administrativo de las Colecciones de la Fundación Miguel Lillo. Actualmente los préstamos de material solo se realizan a investigadores y estudiantes de posgrado (becarios a cargo de investigadores) del interior del país, ya que los préstamos al exterior requieren una serie de engorrosos trámites y documentación para su envío por lo que no se están realizando en estos momentos, pero si se brindan otros servicios como el envío de datos o fotografías que se requieran a través de correo electrónico.

La colección entomológica se encuentra adherida al Sistema Nacional de Datos Biológicos (SNDB) desde el año de su creación (2009), el cual facilita la búsqueda taxonómica y geográfica de especímenes. Desde el año 2002 está incorporada al Global Biodiversity Information Facility (GBIF), momento en el cual se constituyó la Red Nacional de Colecciones (cuya sede está en el Museo Argentino de Ciencias Naturales Bernardino Rivadavia de Buenos Aires) y se designó una Comisión Coordinadora, en la cual participó como miembro la Dra. Mercedes Lizarralde de Grosso, en representación de la Fundación Miguel Lillo. 
En el año 2007, se comenzó a desarrollar una base de datos para uso público sobre especímenes utilizando protocolos formalizados por la Red Interamericana de Información sobre Biodiversidad (IABIN) y el GBIF. Este proyecto dio los impulsos iniciales para la digitalización de datos. Actualmente se está trabajando en la informatización de datos, la cual está en sus inicios, habiéndose digitalizado aproximadamente el $95 \%$ de los ejemplares de la serie tipo, y cerca del $10 \%$ de los conservados en seco y en alcohol. Lo anterior nos permite facilitar la búsqueda de información de los especímenes, así como su manejo y utilización, además de evitar la manipulación innecesaria del material. En el ingreso de datos se utiliza como matriz una base de datos (Access y/o Excel de Microsoft) que contiene diferentes campos (aproximadamente 78) en los cuales se transcribe la información contenida en las etiquetas de cada espécimen, como el código de la colección, número de catálogo, nombre científico, orden, familia, género, epíteto específico, autor, año, país, provincia, localidad, colector, coordenadas geográficas, entre otros.

\section{Convenios y proyectos}

La colección mantiene convenios con otras colecciones o Instituciones, lo cual es sumamente beneficioso, ya que se establecen vínculos formales con otros centros y de este modo se dan las condiciones para incrementar la cantidad y calidad del material de la colección. Tal es el caso del actual "Acuerdo y términos de transferencia de material" entre el Department of Entomology National Museum of Natural History, Smithsonian Institution (Washington D.C.), USA y la FML desde el año 2018. El objetivo de dicho acuerdo es el intercambio de material para el estudio específico de hormigas cultivadoras de hongos que serán utilizadas en el proyecto "Biología de las hormigas cultivadoras de hongos del Piedemonte Andino de Argentina, con énfasis en la especie Paramycetophylax bruchi", a cargo del Dr. Theodore R. Schultz (curador de la colección Hymenoptera del Instituto Stmithsoniano).

La preservación de la colección entomológica está contemplada dentro del proyecto institucional "Preservación, conservación e informatización de la colección entomológica del Instituto de Entomología-FML", diseñado para garantizar su protección, resguardo y adecuada conservación. En él se incluyen las tareas de mantenimiento, monitoreo, reacondicionamiento, diagnóstico, documentación, acrecentamiento, digitalización de datos, adquisición de recursos económicos, especializaciones (cursos, talleres, etc.), seguimiento y publicación de catálogos. Forman parte de este proyecto la curadora, la responsable de la Colección Entomológica Conservada en Alcohol y los técnicos de apoyo, cuya función principal es asegurar el resguardo, conservación, administración de los materiales almacenados y datos electrónicos y propiciar el ingreso de nuevo material y su identificación.

\section{Publicaciones}

La colección entomológica cuenta con numerosos catálogos de insectos publicados tales como: Lista de los tipos de Insecta (Exceptuando Diptera) conservados en el Instituto Miguel Lillo (Hayward, 1963), Los tipos de Diptera conservados en el Instituto Miguel Lillo (Hayward y Golbach, 1963), Lista de los tipos de insectos y otros invertebrados conservados en el Instituto Miguel Lillo (3 $3^{\text {a }}$ entrega) (Hayward, 1967), Lista de los tipos de insectos y otros invertebrados conservados en el Instituto Miguel Lillo (4 ${ }^{\text {a }}$ entrega) (Hayward, 1970), Los ejemplares tipo de Masarinae y Polistinae (Hymenoptera: Vespidae) depositados en la colección del Instituto Fundación Miguel Lillo (IFML) 
(Colomo \& Berta, 2005a), Los ejemplares tipo de Eumeninae (Hymenoptera: Vespidae) depositados en la colección del Instituto Fundación Miguel Lillo (IFML) (Colomo \& Berta, 2005b), Las Dactylopiidae y Diaspididae (Hemiptera: Coccoidea) de la colección Kenneth Hayward, Tucumán, Argentina (Claps et al., 2006), The type specimens of Anthomyiidae, Muscidae and Sarcophagidae (Diptera: Calyptratae) housed at the Instituto- Fundación Miguel Lillo, Tucumán, Argentina (Patitucci et al., 2011), Buprestidae type specimens at Fundación Miguel Lillo, Argentina (MacRae, 2015), los ejemplares de Cercopoidea (Hemiptera: Auchenorrhyncha) depositados en la colección entomológica del Instituto Fundación Miguel Lillo, Tucumán, Argentina (Foieri \& Marino de Remes Lenicov, 2015), Catálogo de los tipos primarios de Coleoptera depositados en la Colección Entomológica del Instituto-Fundación Miguel Lillo (Aranda et al., 2016), Tipos de la familia Cerambycidae (Insecta: Coleoptera) depositados en la Colección Entomológica del Instituto- Fundación Miguel Lillo (Córdoba, 2017), A Catalogue of the type specimens of Heteroptera (Insecta) housed at Instituto Fundación Miguel Lillo (Tucumán, Argentina) (Melo et al., 2018), Catálogo de los especímenes tipo de Ephemeroptera (Insecta) depositados en la colección entomológica conservada en alcohol del Instituto-Fundación Miguel Lillo, Tucumán, Argentina (Córdoba, 2019) y Los ejemplares tipo de Insecta depositados en la colección entomológica conservada en alcohol del Instituto-Fundación Miguel Lillo, Tucumán, Argentina (Córdoba, 2021). Todos ellos con el propósito de mostrar a la comunidad científica el material que resguarda esta colección. Estos trabajos forman parte de los documentos que permiten conocer el material de la serie tipo o del resto de los ejemplares, constituyendo la base de numerosas investigaciones.

\section{Reglamentos}

La administración de la colección entomológica se basa en el Reglamento Administrativo de las Colecciones de la Fundación Miguel Lillo, donde se contemplan las normas sobre los responsables y autoridades, documentación, ingresos y egresos, consultas, préstamos, donaciones, canjes y material tipo. Este reglamento fue puesto en vigencia desde el año 2020, por la Comisión Asesora Vitalicia de la FML, sobre la base del documento elaborado por la Dirección General de Investigación en consulta con la Junta Interna de Asesoramiento Científico y una comisión de investigadores de las áreas científicas, previa consulta a todos los curadores de las colecciones.

\section{Visitas y servicios}

La colección entomológica del I-FML recibe cada año un promedio de 17 vistas de profesionales e investigadores del país y del mundo para consulta de especímenes. También se reciben en promedio 85 estudiantes al año con el objetivo de conocer la colección. Los investigadores son albergados en un espacio especialmente destinado para ellos, el cual cuenta con instrumental óptico y acceso a Internet. Además, cuando no es posible la visita presencial o la consulta puede resolverse de manera remota, se realizan un promedio anual de 30 consultas vía e-mail, teléfono y otras.

El material preservado en la colección ha sido y es permanentemente consultado, a partir del cual se han realizado numerosas investigaciones, tesinas de grado, tesis de maestría y de doctorado. También se realizan servicios de determinaciones a partir de comparaciones de ejemplares obtenidos en el campo con el material depositado en la colección que causan, por 
ejemplo, daños económicos en cultivos o concernientes a la salud (ej. Enfermedad de Chagas, etc.). Existe material separado que se encuentra sin datos de procedencia y es utilizado como material de consulta durante prácticas de cursos de maestría o materias de especialidad.

\section{Perspectivas Futuras}

Como proyecto a futuro, se analiza la posibilidad de incrementar sustancialmente el número de datos digitalizados y georreferenciados, también la creación de una base fotográfica de los ejemplares de la serie tipo. Para ello, se dispone de un microscopio estereoscópico con cámara incorporada (Leica M205 C), que permitirá obtener imágenes de alta resolución y calidad.

Dada la importancia de esta colección, tanto para la comunidad científica como para la sociedad en general, es necesario garantizar la conservación de los ejemplares allí depositados. Para lo cual es imperante y vital destinar un presupuesto que se ajuste a sus necesidades y concretar planes de manejo que aseguren su permanencia en el tiempo, ya que constituye la fuente primaria del desarrollo científico.

AgRAdeCImientos. Deseamos agradecer a Marcela Peralta (Instituto de Invertebrados, FML) y a Hugo Alfredo Carrizo (Instituto de Paleontología, FML) por la revisión y aportes al manuscrito. A María Delia Robles Terán (Dirección de Transferencia y Servicios Externos, FML) y a Francisco Sánchez, José Ortega, Alberto Rojas, Whalter Lemos, Federico Heredia y Rafael Palavecino (Instituto de Entomología, FML) por la información brindada. A la Fundación Miguel Lillo por su constante apoyo y a los Árbitros por sus oportunas sugerencias.

\section{LITERATURA CITADA}

Aranda, S. G., Córdoba, S. P., Sánchez, F. R. (2016) Catálogo de los tipos primarios de Coleoptera depositados en la Colección Entomológica del Instituto-Fundación Miguel Lillo. Acta Zoológica Lilloana, 60 (1), 10-46.

Claps, L. E., Zamudio, P., Díaz Briz, L. (2006) Las Dactylopiidae y Diaspididae (Hemiptera: Coccoidea) de la Colección Kenneth Hayward, Tucumán, Argentina. Revista Brasileira de Entomología, 50 (1), 33-38. https://doi.org/10.1590/S0085-56262006000100004

Claps, L. E., Claps, G. L., Navarro, F. (2013) Abraham Willink: impulsor de la descentralización de la entomología argentina. Revista de la Sociedad Entomológica Argentina, 72 (1-2), 01-13.

Colomo, M. V., Berta, D. C. (2005a) Los ejemplares tipo de Masarinae y Polistinae (Hymenoptera: Vespidae) depositados en la colección del Instituto Fundación Miguel Lillo (IFML), Argentina. Revista de la Asociación Entomológica Argentina, 64 (1-2), 71-84.

Colomo, M. V., Berta, D. C. (2005b) Los ejemplares tipo de Eumeninae (Hymenoptera: Vespidae) depositados en la colección del Instituto Fundación Miguel Lillo (IFML), Argentina. Revista de la Asociación Entomológica Argentina, 64 (3), 23-33.

Córdoba, S. P. (2017) Tipos de la familia Cerambycidae (Insecta: Coleoptera) depositados en la Colección Entomológica del Instituto-Fundación Miguel Lillo. Revista de la Sociedad Entomológica Argentina, 76 (1-2), 61-63. 
https://doi.org/10.25085/rsea.761210

Córdoba, S. P. (2019) Catálogo de los especímenes tipo de Ephemeroptera (Insecta) depositados en la Colección Entomológica Conservada en Alcohol del Instituto-Fundación Miguel Lillo, Tucumán, Argentina. Revista de la Sociedad Entomológica Argentina, 78 (4), 37-54. https://doi.org/10.25085/rsea.780406

Córdoba, S. P. (2021) Los ejemplares tipo de Insecta depositados en la colección entomológica conservada en alcohol del Instituto-Fundación Miguel Lillo, Tucumán, Argentina. Dugesiana, 28 (2), 75-80.

Foieri, A., Marino de Remes Lenicov, A. M. (2015) Los ejemplares de Cercopoidea (Hemiptera: Auchenorrhyncha) depositados en la colección entomológica del Instituto Fundación Miguel Lillo, Tucumán, Argentina. Acta Zoológica Lilloana, 59 (1-2), 57-70. Disponible en: http://www.lillo.org.ar/journals/index.php/acta-zoologica-lilloana/article/view/147 (consultado 23 febrero 2021).

Hayward, K. J. (1963) Lista de los tipos de Insecta (exceptuando Diptera) conservados en el Instituto Miguel Lillo. Acta Zoológica Lilloana, 19, 297-334.

Hayward, K. J., Golbach, R. (1963) Los tipos de Diptera conservados en el Instituto Miguel Lillo. Acta Zoológica Lilloana, 19, 391-406.

Hayward, K. J. (1967) Lista de los tipos de insectos y otros invertebrados conservados en el Instituto Miguel Lillo (3a entrega). Acta Zoológica Lilloana, 12, 337-352.

Hayward, K. J. (1970) Lista de los tipos de insectos y otros invertebrados conservados en el Instituto Miguel Lillo (4a entrega). Acta Zoológica Lilloana, 26, 105-116.

Joffrin, L., Goodman, S. M., Wilkinson, D. A., Ramasindrazana, B., Lagadec, E., Gomard, Y., Le Minter, G., DosSantos, A., Schoeman, M. C., Sookhareea, R., Tortosa, P., Julienne, S., Gudo, E. S., Mavingui, P., Lebarbenchon, C. (2020) Bat coronavirus Phylogeography in the Western Indian Ocean. Scientific Reports, 10, 6873. Disponible en: https://www.nature.com/articles/s41598-020-63799-7.pdf (consultado 03 marzo 2021). https://doi.org/10.1038/s41598-020-63799-7

MacRae, T. C. (2015) Buprestidae type specimens at Fundación Miguel Lillo, Argentina. Disponible en: https://beetlesinthebush.com/2015/03/14/buprestidae-type-specimens-at-fundacionmiguel-lillo-argentina/ (consultado 21 enero 2021).

Melo, M. C., Zamudio, M. P., Dellapé, P. M. (2018) A Catalogue of the type specimens of Heteroptera (Insecta) housed at Instituto Fundación Miguel Lillo (Tucumán, Argentina). Revista de la Sociedad Entomológica Argentina, 77 (2), 1-21.

https://doi.org/10.25085/rsea.770201

Patitucci, L. D., Mulieri, P. R., Mariluis, J. C. (2011) The type specimens of Anthomyiidae, Muscidae and Sarcophagidae (Diptera: Calyptratae) housed at the Instituto- Fundación Miguel Lillo, Tucumán, Argentina. Acta Zoológica Lilloana, 55 (2), 154 -170.

Tobar, D. (2002) Informe de la curaduría de la colección de mariposas "Ernesto Wolfrang SchmidtMumm" del AvH. Bogotá, Colombia.

Thompson, C. W., Phelps, K. L., Allard, M. W., Cook, J. A., Dunnum, J. L., Ferguson, A. W., Gelang, M., Khan, F. A. A., Paul, D. L., Reeder, D. M., Simmons, N. B., Vanhove, M. P. M., Webala, P. W., Weksler, M., Kilpatrick, C. W. (2021) Preserve a voucher specimen! The critical need for integrating natural history collections in infectious disease studies. mBio, 12 (1), 1-20. 
https://doi.org/10.1128/mBio.02698-20

Vargas, H. E. (2010) Monitoreo de humedad y temperatura en colecciones biológicas con herramientas de software libres. Disponible en: https://ahim.wordpress.com/2010/09/21/monitoreo-de-humedad-y-temperatura-encolecciones-biologicas-con-herramientas-de-software-libre/ (consultado 23 marzo 2021). 